\title{
The Design of an Innovative Large Scale Schönflies-Motion Generator
}

\author{
Alexei Morozov and Jorge Angeles \\ Department of Mechanical Engineering \\ $\&$ Centre for Intelligent Machines \\ McGill University \\ Montreal, Canada \\ alexvit@cim.mcgill.ca angeles@cim.mcgill.ca
}

\begin{abstract}
The design of a novel Schönflies-Motion Generator (SMG) is the focus of the paper. Schönflies motions are characterized by four-degrees-of-freedom three independent translations and one rotation about one axis of fixed orientation. The two driving modules of the manipulator are based on differential transmissions. Then, the design philosophy and the layout of the SMG are discussed, along with the design procedure. This procedure includes: (i) part-modelling and visualization, with animation of the device; (ii) evaluation of the main parameters and the characteristics of the different structural realizations, as well as the selection of one single structure meeting best the design specifications; (iii) the design of the main components for the selected variant of the structure; and (iv) the production of detailed manufacturing drawings. Further results of structural and modal analyses of the SMG are considered, while the link geometry is defined based on the design specifications.
\end{abstract}

\section{Introduction}

The need to service, maintain and repair aircraft and other large-scale structures has called for a new generation of manipulators that are characterized by a long reach and a highly redundant architecture [1]. The main problem here is to produce a mechanical system capable of accurate tasks in the presence of a flexible structure. Current designs of such systems exist, but they are limited to tasks that are error-tolerant, e.g., cleaning [2].

In this paper we report on the process of the integrated design and analysis of a novel class of robotic system for Schönflies-motion generation. Such motions comprise three translations and one rotation about one axis of fixed direction. These motions are those produced by what is known as SCARA (Selective-Compliance Assembly Robot Arm) systems, a class of four-degree-of-freedom manipula- tors that exhibit a finite stiffness in four directions of their motion space, while being "infinitely" stiff in the remaining two directions of the space of rigid-body twists [3]. Current manipulators producing this class of motions are most frequently designed with a common architecture, namely, three revolute and one prismatic joints, the latter being indistinctly collocated either at the proximal - to the baseor at the distal joint.

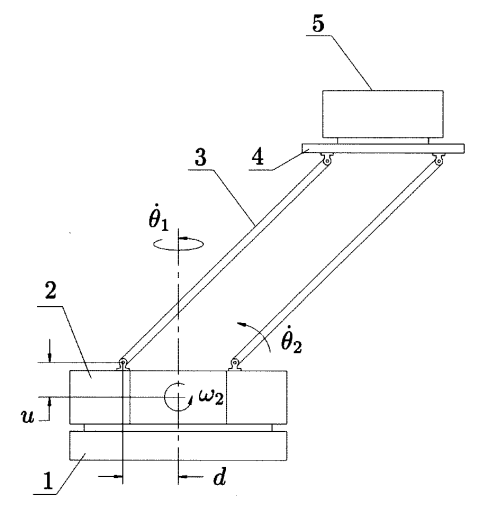

Fig. 1: The pan-tilt motion generator, showing: (1) the fixed base; (2) the rotating housing; (3) the parallelogram linkage; (4) the upper platform; and (5) the payload.

Therefore, a SCARA is uncompliant in two directions of rotation. SCARA systems were originally developed for assembly tasks, hence their name. Any motion with the foregoing features is known also as a Schönflies-motion, after the German geometer Arthur Moritz Schönflies of the 19th century [4], [5], [6], who found that the set has the algebraic structure of a group [7]. For this reason, the set is known as the Schönflies subgroup of the group of rigidbody displacements. The first modern realization of this subgroup was reported by Makino et al. [8], [9], [10], who coined the acronym SCARA. We introduce here a novel architecture producing SCARA motions that is based on 
what is known as a П-joint, as termed by Hervé and Sparacino [11], who introduced the concept concurrently with Wohlhart [12], [13].

To be sure, alternatives to serial architectures of SCARA systems have been proposed: Clavel [14], [15], patented a parallel, three-degree-of-freedom (dof) robot capable of producing pure translations of its moving platform. By adding, in series, a fourth controlled axis, the hybrid architecture thus resulting becomes a SMG. More recently, Company et al. [16], [17], patented a fully parallel SCARA system, as yet to be put to work in production environments.

\section{Design Philosophy}

The design philosophy adopted at the outset hinges upon simplicity. Besides simplicity, the design is driven by the performance specifications, of course, as any design task. The design specifications are described in detail in Section 3, that relevant at the preliminary stage being long reach: The manipulator must be capable of accessing all points of the fuselage of an aircraft of any size, including wide-body airplanes.

Simplicity is broadly recognized by designers the world over as the cornerstone of any design task. Hence, one might think that simplicity is intuitively apparent and one should not have any problem with applying the concept at the early design stages. Problems arise when one attempts to measure the simplicity or, equivalently, the complexity of a design. Indeed, attempts to measure complexity have been reported in the design literature. For example, the first axiom proposed by Suh [18] postulates that the best design is one with the minimum information content. Suh goes on to quantify information content of a design as the binary logarithm of the probability of meeting the functional requirements, or design specifications. The problem now is how to assign a probability in this context. El-Haik and Yang [19] resorted to Suh's axiomatic design to define design complexity in terms of three concepts: coupling; variability; and correlation. ElMaraghy and Urbanic [20] associated diversity to complexity, while adopting Suh's axiomatic design as well. Diversity can be thought of as synonimous with variety in the two foregoing works.

As a means to measure design complexity, one can resort to Kolmogorov's complexity [21], [22] which, in principle, can be applied to measure the complexity of any object. The fact of the matter is, as Delahaye [23], [24] recognizes, Kolmogorov's complexity has been applied only to abstract domains of science and, to the authors' knowledge, not yet to design. Delahaye even points out serious philosophical problems in connection with Kolmogorov's complexity, that touch upon the very foundations of mathematics, namely, Gödel's incompleteness results [25].

Nevertheless, engineering design, or engineering at large for that matter, must deliver even before a sound theory is available to support the engineer's judgment when it comes to decision-making. We thus propose here, as a measure of complexity in the context of robot preliminary design, the diversity of components performing functions of the same family. In this regard, we postulate that, for the design of a Schönflies motion generator, complexity grows with diversity. More specifically, we look at the actuation subsystem of a SMG: The simplest SMG is one with its four actuators identical. We have three alternatives:

(a) Serial architectures: The actuators are collocated at each of the four independent joints.

(b) Hybrid architectures: Some actuators are placed in series, some in parallel.

(c) Parallel architectures: All four actuators are placed on one common base.

Serial architectures, the most common ones in industrial SCARA systems, exhibit a concatenation of actuators, from shoulder to wrist, with an intermediate elbow joint. Moreover, the wrist has a fourth joint, prismatic, in series with its revolute joint.

One example of hybrid architecture is that of the Delta robot [14], [15]. This robot features a parallel architecture that allows three-dof pure translations of its moving platform. The three motors moving this platform are placed on a fixed base, usually the ceiling, with the three motor axes forming an equilateral triangle. Mounted on the same platform is a fourth actuator, providing the rotation about an axis normal to the plane of the first three actuators.

Parallel architectures are not as yet available commercially, but two instances can be cited: The H4 Robot [16], [17] and that disclosed in a patent application filed by the authors [26]. In these two designs, the four actuators are placed at the base.

In terms of diversity, it is apparent that serial architectures offer the richest, and hence, the most complex architecture. At the other end of the spectrum, parallel architectures offer the simplest alternative. In-between we have the hybrid architectures.

Based only on complexity, we would choose a parallel architecture. However, we must also consider workspace volume, for the tasks targeted require a long reach and high maneuverability, which are not possible with parallel architectures. We are thus left with serial and hybrid architectures, which are discussed in Subsection 3.1. 


\section{Architecture of the Schönflies-Motion Generator}

As per our main design specification, the manipulator under design should be capable of producing three independent translations and one rotation about a vertical axis. The end-effector (EE), which is also referred to as the endplatform, or end-plate, thus undergoes Schönflies displacements. The SMG, built as a laboratory prototype of a largescale manipulator for servicing large-scale structures, such as aircraft fuselages, civil structures, etc., is intended for the translation of the base of a robot along the prescribed trajectory [27]. To this end, the horizontal reach of the SMG should be $2.5-3.0 \mathrm{~m}$, and vertical reach of 3.5-4.0 m.

Our aim here is a testbed where a large robot with flexible links carries a smaller robot with rigid links on its end platform. In order to allow for the testing of robot control algorithms that compensate for the motion of the base of the smaller robot, the flexible robot should be capable of being excited by the inertia forces of its smaller counterpart. These forces being associated with rigid links are expected to have predominantly low frequency components, within the 5-Hz bandwidth. Then, flexible displacements of the end-platform should be compensated online by the control system. The flexible robot should be able to undergo flexible translations vertically and horizontally, while showing a high torsional stiffness. The purpose of this structure is to keep the end plate as horizontal as possible.

We have decided to make the SMG as structurally flexible as possible in order to decrease its first two natural frequencies to the specified bandwidth, thus enabling compensation of the resulting deformations of the large robot- the SMG - online by means of the motors of the smaller sevendegree-of-freedom (seven dof) robot. However, the design of the smaller robot lies beyond the scope of this paper.

\subsection{Architecture Alternatives}

From the above-mentioned specifications, the SMG was designed with a reach of $3.0 \mathrm{~m}$, as the first module of an 11-dof system for highly demanding tasks in terms of positioning accuracy, e.g., stripping and painting of aircraft fuselages.

In our design [28], the actuation of the manipulator is based on a cascade of two functionally identical tilt-pan motion generators, each providing one rotation about a horizontal axis, the tilt, and one about a vertical axis, the pan, while keeping the top link horizontal as depicted in Fig. 1.

In our design we considered two different architectures of the manipulator, the serial and the hybrid.

In the hybrid architecture, and with the aim of generating two independent motions of tilt-pan mechanisms with substantially different load conditions by means of identical motors, we decided to use a differential gear train. This train admits as inputs the motions of two identical DC motors, their outputs providing for a pan motion about a vertical axis, at a rate $\dot{\theta}_{1}$, and a tilt motion about a horizontal axis, of a rate $\dot{\theta}_{2}$. In the hybrid layout, two motors in each pan-tilt mechanism are set in a parallel position, while the two mechanisms are laid out in series.

However, the hybrid manipulator is expensive to manufacture because of the use of more complicated and costly components of the gearing system. In order to reduce the manufacturing cost we decided to design a symplified version of the SMG where all four motors are in series. In this case we avoid the expensive differential gear trains, although we still need gear reducers.

Finally, both architectures-hybrid and serial-were developed; however, only the serial solution was within our budget, and hence, was the only one manufactured.

\subsection{The Pan-Tilt Mechanism}

The hybrid architecture being the more innovative of the two, we expand on its design in this paper. In this architecture, the pan motion, represented by angle $\theta_{1}$, is generated directly; the tilt motion, represented by angle $\theta_{2}$, however, is generated indirectly, by means of a mechanism that transmits the angular velocity $\omega_{2}$ of the horizontal output shaft of a differential gear train to a parallelogram, as shown in Fig. 1.

A $\Pi$ joint $[11]$ is used to produce a relative translation of the two coupled links along a circular trajectory. Such a joint is a parallelogram mechanism, the two coupled links being the base and the coupler links of the underlying fourbar linkage. We use a concatenation of two such joints to produce two independent translations in corresponding vertical planes. Each vertical plane is rotated about a vertical axis, the first of them by means of a revolute joint with vertical axis fixed to the base of the manipulator; the second plane is rotated about a vertical axis fixed to the top link of the first parallelogram.

\subsection{Forward and Inverse Kinematics}

The Cartesian variables of the manipulator are four, namely, the three components of the position vector $\mathbf{p}$ of the operation point $P$ of the end-plate (EP), and angle $\phi$, as shown in Fig. 2. If we let

$$
c_{i} \equiv \cos \theta_{i}, \quad c_{i j} \equiv \cos \left(\theta_{i}+\theta_{j}\right)
$$

with similar definitions for $s_{i}$ and $s_{i j}$, then the link-length projections $\lambda_{1}$ and $\lambda_{2}$ indicated in this figure on the $X-Z$ 

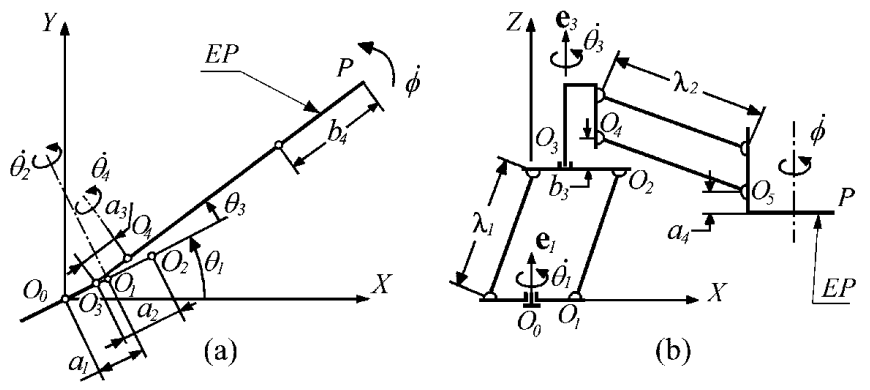

(b)

Fig. 2: The kinematic chain of the SMG: (a) top view; (b) side view

plane are defined as:

$$
\lambda_{1}=l_{1} \sqrt{1-s_{1}^{2} c_{2}^{2}}, \quad \lambda_{2}=l_{2} \sqrt{1-s_{13}^{2} c_{4}^{2}},
$$

with $l_{1}$ and $l_{2}$ denoting the link lengths of the lower and upper parallelograms, respectively. The forward kinematics relations take the form

$$
\begin{aligned}
& x=\left(a_{1}+l_{1} c_{2}-a_{2}\right) c_{1}+\left(a_{3}+l_{2} s_{4}+b_{4}\right) c_{13}, \\
& y=\left(a_{1}+l_{1} c_{2}-a_{2}\right) s_{1}+\left(a_{3}+l_{2} s_{4}+b_{4}\right) s_{13}, \\
& z=l_{1} s_{2}+b_{3}-l_{2} c_{4}-a_{4}, \\
& \phi=\theta_{1}+\theta_{3} .
\end{aligned}
$$

in which $x, y$, and $z$ denote the three components of $\mathbf{p}$ in the base coordinate system $X, Y, Z$ of Fig. 2.

The inverse kinematics of this manipulator [3] admits two postures for a given pose of the end-plate. As the reader can readily visualize from Fig. 2, these two postures are elbow-up and elbow-down, which are present in manipulators with a sagital plane of symmetry.

\section{The Differential Mechanism}

With the aim of generating two independent motions in the pan-tilt mechanism with substantially different loading conditions - one motion takes place in a vertical plane; the other in a horizontal plane - by means of identical motors, we decided to use a differential gear train that would admit as inputs the motions of two identical DC motors, their outputs providing for a pan motion about a vertical axis and a tilt motion about a horizontal axis. This mechanism is shown in Fig. 3. In this figure we have: the fixed base (1); the support of the differential unit and internal gearing (2); the differential gear train (3); the rotating housing (4); the output shafts; and (6) output reversing unit (5).

The main features of the differential transmission are: both motors are installed on the base; the input shafts are

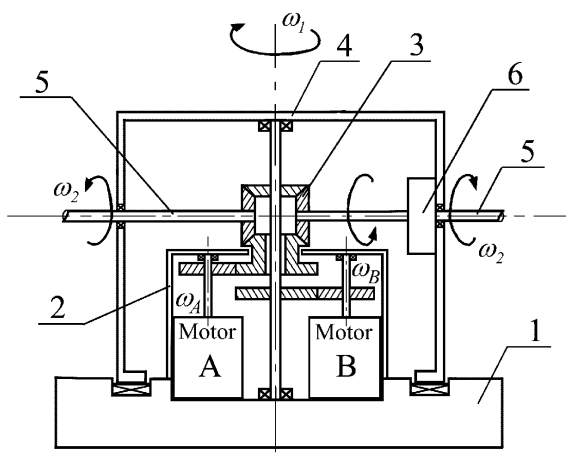

Fig. 3: The two-dof drive with its differential unit.

coaxial; the housing of the module is the case of the differential; and one of the output horizontal shafts should have a reverser to provide identical rotations of these shafts.

Let us denote with $\omega_{A}$ and $\omega_{B}$ the input velocities of the differential unit, i.e., the velocities of motors $\mathrm{A}$ and $\mathrm{B}$, the velocity of the planet gears of the differential unit around their axes being $\omega_{2}$ and $-\omega_{2}$, by design. Moreover, the vertical component of the angular velocity of the planet gears, i.e., the velocity of the planet-carrier, is denoted by $\omega_{1}$.

The rotations from motors A and B are transmitted to the lower and upper sun gears of the differential unit via intermediate gearing. The differential gears are arranged in such a way that the two input shafts of the differential unit are coaxial, and both driving motors are located below the differential. For this purpose we use one hollow shaft, traversed by a second shaft. This design makes it possible to keep both motors on the base and to achieve a free rotation of the housing around the vertical axis.

Notice that, if $n_{A}=n_{B}=n$ and $N_{S}=N_{P}$, then the foregoing relations become

$$
\omega_{1}=\frac{n}{2}\left(\omega_{A}+\omega_{B}\right), \quad \omega_{2}=\frac{n}{2}\left(\omega_{B}-\omega_{A}\right),
$$

i.e., the panning is proportional to the mean value of the angular velocities of the two motors, while the tilting $\dot{\theta}_{2}$ is proportional to their difference. This means that this layout can achieve a slow tilting with high motor angular velocities, a desirable feature to rise heavy loads.

The horizontal shafts of the planet gear stand out of the housing of the drive, to which they are coupled by means of bearings. When the planets rotate in a horizontal plane about the central vertical axis, the housing follows the planets and rotates about the same axis with a velocity $\omega_{1}$. The output velocities $\omega_{1}$ and $\omega_{2}$, in turn, are related to the input velocities $\omega_{A}$ and $\omega_{B}$ as shown below:

$$
\omega_{1}=\frac{n_{A} \omega_{A}+n_{B} \omega_{B}}{2}, \quad \omega_{2}=\frac{\left(n_{B} \omega_{B}-n_{A} \omega_{A}\right)}{2} \frac{N_{S}}{N_{P}},
$$


where $n_{A}$ is the reduction ratio of the gear train between motor A and the shaft of the first sun gear, with a similar definition for $n_{B}$. Moreover, $N_{S}$ is the tooth number of the sun gears, and $N_{P}$ is that of the planet gears.

Now, the output shafts rotate in the opposite directions. As a result, after turning these shafts upward, from the top of the module, we will see rotation of both shafts in the same direction, which poses a design challenge in producing the tilt motion of the parallelogram linkages. To face this challenge we need to implement an output-reversal on one of the shafts. This will provide synchronous opposite rotation of the driving shafts turned upwards, thus enhancing the static stability of the system.

A simplistic solution to the above problem would be to use the torque from only one of the two planets, but this idea is rejected because it destroys the symmetry of the design. Indeed, under this solution, only one side of the housing is loaded, which brings about significant bending moments that can produce collapse.

Another solution would be to transmit the two opposite torques of the planet shafts to a common horizontal shaft. This solution, however, requires an offsetting of the common shaft with respect to the pan axis, in order to avoid interference with the shaft of the upper sun gear, and is equally discarded. The offset will produce significant bending moment on the housing.

\section{Design Criteria and Layout}

First and foremost, our design of the pan-tilt unit allows for a virtually unlimited variety of link arrangements, as illustrated in Figure 4: Fig 4(a) shows the parallelogram linkage with a horizontal layout of its pin-joints; Figure 4(b) depicts a vertical layout of the joints; in Fig. 4(c) the layout is inclined at an angle $\alpha_{p}$, which can take a broad range of values, depending on specific requirements of the manipulator.

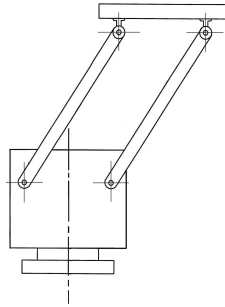

(a)

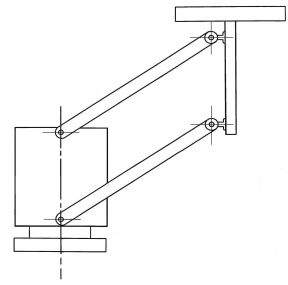

(b)

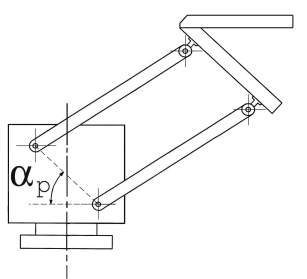

(c)
Fig. 4: Various layouts of the pin joints for the tilt motion: (a) horizontal; (b) vertical; (c) inclined

In our design of a laboratory prototype we selected the horizontal layout of the pin-joints: for the lowerproximal-module; for the upper-distal-module a vertical layout.

Now, contrary to the pan motion, the tilt motion can be produced in different ways. The output rotation of the differential horizontal shaft can be transmitted to the parallelogram linkage by means of ball-screws, rack-and-pinion transmissions, gear reducers, worm-gears, etc.

At the outset, we imposed four main design criteria, as described below:

First, the linkage and housing driving system must have a self-locking capability, a feature which is imperative for any industrial robotic system for safety reasons. This feature can be realized using brakes or any other means.

Second, we should achieve as much load-balance and mechanism simplicity as possible. Load-balance is intended for a uniform distribution of the load on the motors and the structure. Simplicity is indeed needed, but it transcends the mechanical system and touches also upon the control algorithms to be developed. If we introduce a mechanism with a non-uniform velocity ratio, the control algorithm will be more complex due to the consequent increase in the complexity of the underlying Jacobian matrix. This will undoubtedly make the optimization of the whole system more cumbersome. Simplicity thus relates to both the mechanical design and the control algorithm.

The third design criterion is reliability, which follows directly from the first two. The simpler the mechanism layout, the less parts the mechanism has, which implies a lower likelihood of part failure, and hence, a higher reliability.

Finally, the fourth criterion pertains to the interchangeability of links with different elastic characteristics. The SMG being intended for a broad variety of tasks, a rich spectrum of operating frequencies must be accomodated. For this reason, we designed the system with interchangeable links at all four parallelograms.

Besides these main criteria we also considered several others, which need not be given the highest priority, but still are relevant. For example, we are not restricted by the compactness of the system, which will rest on the ground, occupying a reasonably large space. However, the upper tilt-pan module should be lighter, and smaller than the first (proximal to the ground) module because of stability reasons (in order to keep the center of mass as low as possible.) Besides, the workspace of the distal module must not intersect that of the proximal one, which brings about additional constraints.

We considered different possibble layouts of the SMG, which can be grouped according to the main types of link drives used: ball-screw transmissions; rack-and-pinion and slider transmissions, as depicted Fig. 5; gear train transmissions; and worm-gear transmissions. 


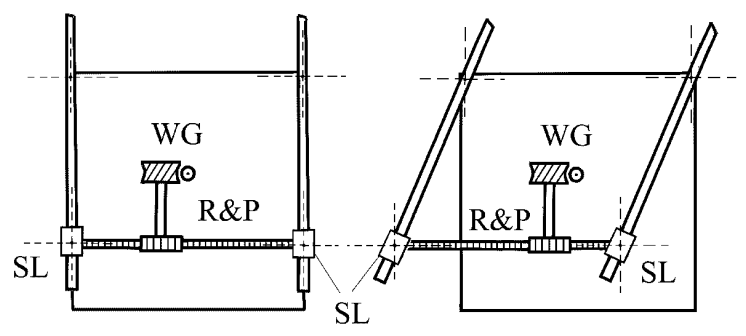

Fig. 5: Layout of the SMG driven by rack-and-pinion transmissions, showing: the worm-gear WG, the rack-andpinion R\&P, and the slider SL: (a) home configuration; (b) arbitrary configuration

Because belt-pulley and chain-sprocket transmissions are marred with bulkiness, backlash, friction, flexibility, inaccuracy and high level of noise, we discarded them at the outset.

Rack-and-pinion transmissions, Fig. 5, can do the job, but they need one inversor on one of the output shafts in order to provide similar direction of motion for the racks of the opposite sides. These transmissions also can produce only non-uniform velocity transmissions, and need extra space for the driving rack with the lower ends of the linkages.

Yet another possible layout for the tilt motion is shown in Fig 6(a). Here, the output rotation of the differential is transmitted directly to the tilt motion of the parallelogram linkage. However, in this case, as in others, we need to synchronize the motion of the output shafts. That is why the opposite side of the module should have an inversor, as shown in Fig 6(b).

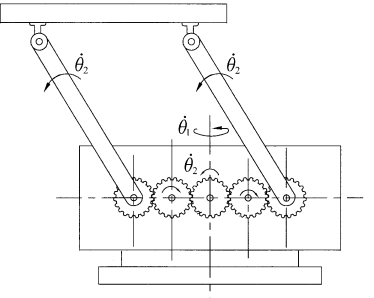

(a)

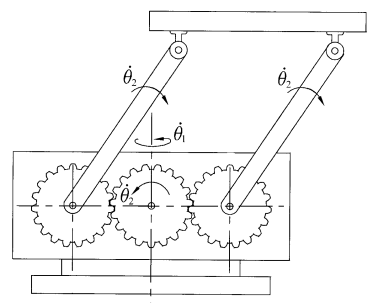

(b)
Fig. 6: Alternative realization of the tilt motion: (a) direct gear transmission for the tilt motion; (b) gear transmission for tilt motion with inverter.

The most interesting layouts are those with ball-screws and worm-gear transmissions. The design on the basis of the above criteria led us to developing these two main transmission layouts, both of which meet the design specifications.

\subsection{Ball-Screw Drives}

Different designs of pan-tilt mechanisms based on ballscrews were analyzed. Here we describe the most typical
SMG of this kind, designed with two ball-screws in combination with two pairs of universal joints, transferring the rotation of the differential output shaft from a horizontal axis to a vertical axis, as shown in Fig. 7. In this figure: the fixed base (1); the housing with differential unit (2); the driven linkage of the tilt-pan mechanism (3); the upper platform (4); the ball-screw shafts (5); the universal joint pair (6); and the ball-screw nut (7).

With this layout we can obtain a self-locking capability of the driving system, if proper parameters of the ball-screw drives are selected. At the same time this layout is characterized by a non-uniform velocity transmission between the differential output $\omega_{2}$ and the parallelogram linkage angular velocity $\dot{\theta}_{2}$.

The conversion of the translational velocity $\dot{s}$ of the ballscrew nut along the ball-screw shaft into the angular velocity $\dot{\theta}_{2}$ of the driven link takes the form

$$
\dot{\theta_{2}}=\theta_{2}^{\prime}(s) \dot{s},
$$

where $\theta_{2}^{\prime}(s)=\mathrm{d} \theta_{2} / \mathrm{d} s$. Moreover, angle $\theta_{2}$ can be found from the geometry of the mechanism, which yields the relation

$$
\begin{aligned}
& \frac{s^{2}-u^{2}-(d+r)^{2}}{2 r} \tan ^{2} \frac{\theta_{2}}{2}-2 u \tan \frac{\theta_{2}}{2} \\
& +\frac{s^{2}-u^{2}-(d-r)^{2}}{2 r}=0,
\end{aligned}
$$

whence

$$
\theta_{2}^{\prime}(s)=\frac{1}{r} \sin \left[\arccos \left(\frac{r^{2}+s^{2}-h^{2}-d^{2}}{2 r s}\right)\right] .
$$

Moreover, the velocity $\dot{s}$ is linearly related to the output velocity $\omega_{2}$ of the differential mechanism via the lead $\lambda$ of the ball screw:

$$
\dot{s}=\lambda \omega_{2},
$$

thereby completing the instantaneous kinematics of the proximal tilt-pan mechanism. The corresponding relations for the distal mechanism follow exactly the same pattern, if with minor modifications.

Apparently, this layout entails a rather cumbersome velocity relation, besides containing two ball-screw drives and two pairs of universal joints connecting the differential outputs with the ball-screw shafts.

Another important parameter should be taken into account when considering ball-screws. This parameter is the transmission angle, or its generalization, the transmission index introduced in [29]. In Fig. 7(a) the transmission angle $\mu$ can be seen as the angle between the driving link 3 of the parallelogram of the SMG and the ball-screw shaft 5. It is apparent that the angle here can be too small, and hence, lead to a poor transmission. To make the angle $\mu$ 
bigger it is necessary to increase the dimension of the base 2 in comparison with the distance between the joint of the link 3 on the base 2 and the joint connecting link 3 with the nut 7 . We need also to increase the distance between the pair of universal joints 6 of the ball screw shaft 5 and the current position of the nut 7 . Such a ratio will provide a value of $\mu$ closer to $\pi / 2$, and a transmission index closer to 1 . However, the angle $\mu$ should not exceed $\pi / 2$ by too much, because $\mu$, approaching $\pi$, is the same as $\mu$ approaching zero; the transmission index in this case would also approach zero.

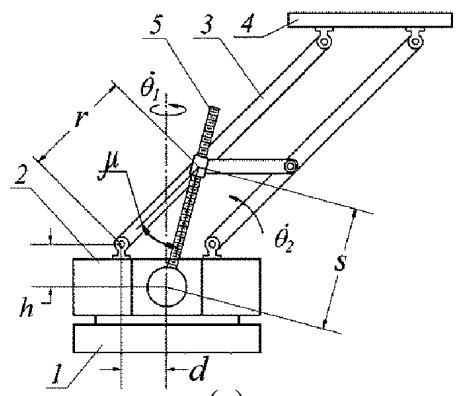

(a)

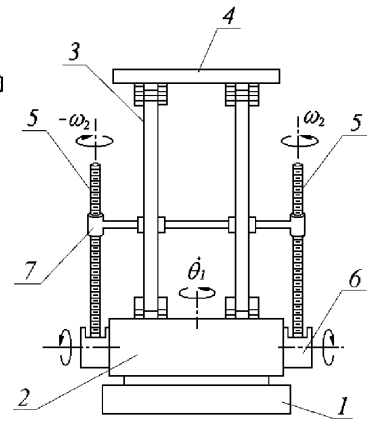

(b)
Fig. 7: The pan-tilt mechanism with ball screws: (a) a side view; (b) a front view.

Thus, the problem of selection of a proper transmission angle is one of optimization of the ratio between the dimension of the base and the lengths of the driving link of the parallelogram and the ball-screw shaft.

In our case, however, the ability to increase the base is restricted because this will lead to increasing the overall size of the manipulator, which is not critical, but is not desirable. We are also restricted in displacing the pin joint of the driving link farther from the joint of the ball-screw shaft because of the same design limitations. As an alternative to enlarging the base we can decrease the lengths of the link 3 and the shaft 5 . However, this solution will lead to a smaller driving lever, and for the same driving moment the drive will have to produce a bigger force, i.e. the drive itself should be more powerful, which means that the mechanism will become bulkier and more expensive, although the transmission index may be much higher.

From the above discussion it should be apparent that with a ball-screw transmission it is possible to provide a good transmission angle only at the cost of bigger dimensions and an increase of power and cost of the drive system.

\subsection{Worm-Gear Drives}

A second layout of the pan-tilt mechanism has two worm-gears. They reduce the angular velocity $\omega_{2}$ of the differential output to produce the angular velocity $\dot{\theta}_{2}$ of the lower parallelograms, which, in turn, transfer the output rotations of the worm gears to the motion of the linkage parallelograms, as shown in Fig. 8.

The driving system of the proximal module is characterized by the features described below: The lower parallelograms (LP) are kept on both sides of the housing of this module. The rotation of the output shafts of the worm-gears (WG output) is directly transmitted to the upper parallelograms (UP). As a result, the velocity ratios of both the worm-gear outputs and the driven links in this layout are constant, thereby allowing uniformity of transmission and simple control algorithms. It is noteworthy that the lower parallelograms do not interfere with the upper ones; however, a ball-screw shaft, with its free end protruding as depicted in Fig. 7, might cause interference.

Finally, the direct motion transmission between parallelograms leads to a simpler and more reliable structure, for neither ball-screws nor universal joints are needed.

The distal module is even simpler and does not need intermediate parallelograms between worm-gears and linkage parallelograms. It is apparent that both proximal and distal modules in this layout bear similarities. The first similarity is that worm-gears allow for a high gear ratio, thus doing away with extra gears in the motor, while reducing mass and volume. The second is a reliable self-locking mechanism. Moreover, by selecting worm-gears with op-

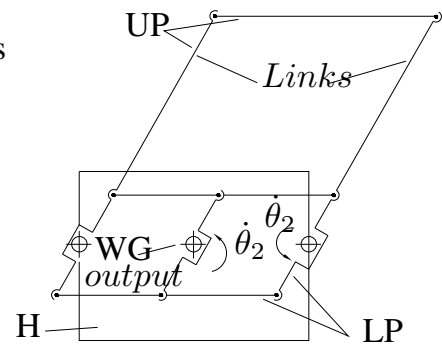

Fig. 8: The layout of choice: the drive system based on worm gears

posite hands, the velocity inversor is eliminated.

We can thus conclude that the two above-mentioned layouts-with ball-screw drive and with worm-gear drive-provide self-locking capability and are statically well balanced by virtue of the two parallelograms in each linkage. At the same time both design solutions need an inversor on the output of one of the two tilt shafts in each module. Besides these features, the ball-screw based layout is marred by a non-uniform velocity ratio; it also needs additional means for turning the angular-velocity vector of the output shafts from a horizontal direction into a vertical direction. The worm-gear solution provides a 
uniform velocity transmission.

From the foregoing analysis of alternatives it becomes apparent that the best solution would be the worm-gear layout.

Regarding the question of self-locking, a third, simplified, solution is always possible, namely, one based on powerful brakes; this may allow us to eliminate the wormgears and to reduce significantly the weight of the structure, which in turn may lead to a lower manufacturing cost.

\subsection{Schönfy-S: Budget-driven Solution}

We designed several types of worm-gear-based SMG. The one described in this paper, with differential gear train inside, is a hybrid, parallel-serial manipulator, as we have in each of the two serial modules two identical motors in parallel. However, for purposes of laboratory testing of link behavior and robot dynamics, we had to go with a serial version of the SMG in order to stay within budget; in this version, all four motors are collocated one after the other. The cost we had to pay for this choice is load-balancing. The prototype of the final design of the serial SMG, dubbed Schönfy-S, is shown in Fig. 9.

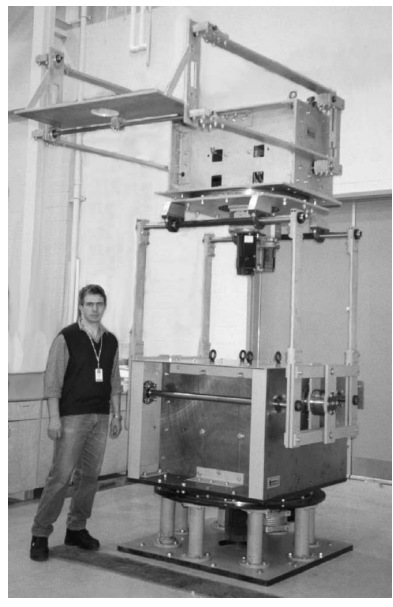

(a)

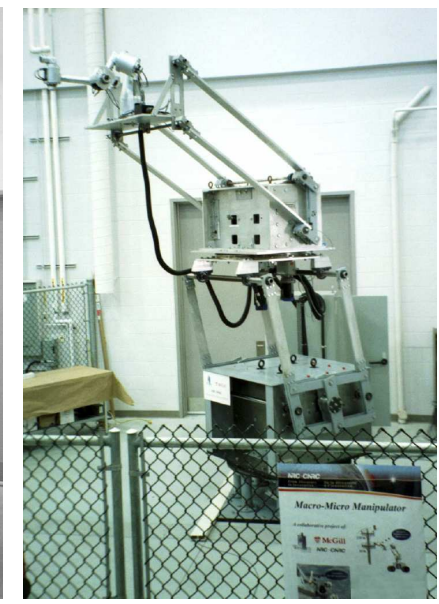

(b)
Fig. 9: Schönfy-S, the laboratory prototype of the serial SMG: (a) with the first author beside (height $=1.8 \mathrm{~m}$ ); (b) with a smaller robot installed on the end platform.

The design involving two pairs of identical motors and two differential gear trains, dubbed Schönfy-H, is a hybrid parallel-serial SMG. While this design meets better our performance functions than its serial counterpart, it turned out to be twice as expensive to manufacture. We are currently working on a parallel SMG, dubbed Schönfy-P.

The main features of Schönfy-S are: maximum vertical reach, $3.8 \mathrm{~m}$; maximum horizontal reach, $2.7 \mathrm{~m}$; minimum vertical reach, $1.4 \mathrm{~m}$; minimum horizontal reach, 0.45 m; weight, 30,000 N. In this robot worm-gears for high transmission ratio were used. The speed ratios and motion ranges of both modules of the serial SMG are shown in Table 1 .

\begin{tabular}{|c|c|c|c|c|}
\hline & PM $N_{\operatorname{Max}}$ & $\mathrm{PM} R_{m}$ & $\mathrm{DM} N_{\operatorname{Max}}$ & $\mathrm{DM} R_{m}$ \\
\hline Pan & $2.0 \mathrm{rpm}$ & $\pm 60^{\circ}$ & $4.0 \mathrm{rpm}$ & $\pm 80^{\circ}$ \\
\hline Tilt & $2.0 \mathrm{rpm}$ & $\pm 38^{\circ}$ & $3.0 \mathrm{rpm}$ & $\pm 40^{\circ}$ \\
\hline
\end{tabular}

Table 1: Maximum speeds $N_{\text {Max }}$ and ranges $R_{m}$ of the pan and tilt motions of the Schönfy-S modules, where PM stands for Proximal Module and DM stands for Distal Module.

The maximum payload on the end-platform of the SMG is $700 \mathrm{~N}$.

\section{General System Design}

The general design procedure for the SMG was divided into five main steps: (i) simulation and visualization of the whole system and its operation before prototyping; (ii) estimation of the main features of the two competing layouts and selection of the one most suitable for the specifications; (iii) designing the main units and the components of the selected layout; (iv) dimensioning the physical parameters of the manipulator for mathematical modeling, namely, its inertial and elastic properties, to conduct elastostatic and elastodynamic analyses for verification of the strength of the individual components and modal analysis; and (v) developing detailed manufacturing drawings.

The design procedure can also be describeded by four different design levels:

1. Simulation and visualization of the overall manipulator. Here we derived the general kinematics of the mechanism and produced a visualization of its workspace. This level consists in turn, of two main stages: (i) simulation of the overall mechanism; animation; expert appraisal of efficiency and workspace; preliminary analysis of forces, torques, inertial parameters, and speeds and accelerations of the system; and (ii) a detailed modeling of the driving system, the main purpose being to elaborate a principal design and to develop the animation of the drives; in this stage we also used expert appraisal of the design by a manufacturing engineer, Technospin Inc.'s Mr. J. Slanik, and conducted the preliminary kinetostatic analysis of the driving system.

2. Simulation of the drive system in full detail, besides the calculation and selection of custom-made and off- 
the-shelf components. This level resulted in the detailed design of the drive system, which became the skeleton of the design, to which all other components and units were attached. One of the most important steps in this level is the complete dimensional design of the whole system.

As indicated earlier, the first two levels of the design resulted in developing systems with two different drive mechanisms: with ball-screws (Fig. 7), and with worm-gears (Fig. 8).

The analysis conducted on the principles of the criteria, developed during the design work and described earlier, has shown that the design of Fig. 8 was better than that of Fig. 7.

3. After selection of the suitable layout, modelling all components and assembling them into the mechanism, their inertial properties had to be verified and corrected on the third level of the design, structural analysis. The main purpose of this level is material selection via the structural analysis of custom-made components. In this stage the design solutions are verified, including adjustments of wall thickness, hole diameters, weight savings, strengthening with ribs, verification of material properties, material reselection, etc.

All these procedures are iterative, until the best combination is achieved. The engine driving the iterations is expert structural engineering knowledge, which is then verified with finite element analysis.

A stress analysis of the units designed and their components, while taking into account material selection and part shapes, led to significant improvements of the initial design. We shall describe presently the stress analysis of one of the critical components.

4. The last level, finalizing the whole process prior to manufacturing, is the preproduction corrections. This level is devoted to issuing manufacturing drawings and to adjusting design details in terms of manufacturing processes. This level is related not with the functional features of the design, but rather with manufacturing standards and technological processes, including materials blank dimensions available in the market. For example, this step may demand from the designer to change some parameters of the components in order to tie them to standard aluminium or steel blanks used for manufacturing parts.

It is noteworthy that all design levels are followed by necessary corrections, which brings about feedback loops in the design process.

\section{Conclusions}

The design of a novel four-dof manipulator for the production of Schöenflies displacements was discussed in this paper. The novelty of the design lies in the use of two similar mechanisms in a serial array, each designed to produce a two-dof motion of a plate, the overall mechanism thus providing a four-dof motion. The end-plate can be used to support either a dead load to be manipulated, for example, in pick-and-place operations, or another manipulator to perform fine-posing tasks. The structure of the manipulator is designed and modelled in Pro/ENGINEER, under the main criteria: simplicity, reliability, and safety. The kinematics of the manipulator and the design procedure were discussed. Simulations were conducted to support the design decisions reported herein, which were then verified on the physical prototype.

\section{Acknowledgements}

The work reported here was supported by NSERC (Canada's Natural Sciences and Engineering Research Council) under Strategic Project 215729-98. The work of Lama Al Bassit, an exchange student from Université de Paris 6, is dutifully acknowledged.

\section{References}

[1] Hiller, M., (1996). "Modelling, simulation and control design for large and heavy manipulators", Robotics and Autonomous Systems, Vol. 19, No. 1, pp. 167-177.

[2] Schraft, R.D., and Wanner, M.C., (1993). "The cleaning robot 'Skywash', Industrial Robot, Vol. 20, No. 6, pp. 21-24.

[3] Angeles, J., (2002). Fundamentals of Robotic Mechanical Systems. Theory, Methods, and Algorithms, Springer-Verlag, New York, 2nd Edition.

[4] Schönflies, A.M., (1886, 1887). "Über Gruppen von Bewegungen”, Math. Ann., Vol. 28, pp. 319-342, \& Vol. 29, pp. 50-80.

[5] Schönflies, A.M., (1889). "Über Gruppen von Transformationen des Raumes in sich", Math. Ann., Vol. 34, pp. 172-203.

[6] Bottema, O., and Roth, B. (1979). Theoretical Kinematics. New York: Dover Publications, pp. 312-315. 
[7] Hervé, J.M., (1978). “Analyse structurelle des mécanismes par groupes de déplacements", Mechanism and Machine Theory, Vol. 13, pp. 437-450.

[8] Furuya, N., and Makino, H., (1980). "Research and development of selective compliance assembly robot arm. I. Characteristics of the system", Journal of the Japan Society of Precision Engineering/Seimitsu Kogaku Kaishi, Vol.46, No.12, pp. 1525-1531.

[9] Makino, H., and Furuya, N., (1982). "SCARA robot and its family", Proc. 3th International Conference on Assembly Automation and 14th IPA Conference, Stuttgart, West Germany, 25-27 May 1982, Kempston, UK, pp. 433-444.

[10] Makino, H., and Furuya, N., (1981). "Motion control of a jointed arm robot utilizing a microcomputer", Proc. 11th International Symposium on Industrial Robots, Tokyo, pp. 405-412.

[11] Hervé, J.M., and Sparacino, F., (1992). "STAR, a new concept in robotics," Proc. 3rd Int. Workshop on Advances in Robot Kinematics, Ferrara, September 79, pp. 176-183.

[12] Wohlhart, K. (1991). "Der homogene ParalleltriebMechanismus," Mathematica Pannonica, Vol. 2, No. 2, pp. 5976.

[13] Wohlhart, K. (1992). "Displacement Analysis of the General Spatial Parallelogram Manipulator," Proc. 3rd International Workshop on Advances in Robot Kinematics, Ferrara, Italy, pp. 104111.

[14] Clavel, R., (1988). "Delta, a Fast Robot with Parallel Geometry," Proc. 18th Int. Symp. Industrial Robots, Lausanne, pp. 91-100.

[15] Clavel, R. et al. (1990) Device for the Movement and Positioning of an Element in Space. US Patent, US4976582, December 11, 1990.

[16] Krut, S., Company, O., and Pierrot, F. (2002) "Velocity Performance Indexes for Parallel Mechanisms with Actuation Redundancy," Workshop on Fundamental Issues and Future Research Directions for Parallel Mechanisms and Manipulators, Quebec City, Canada, October 2-4.

[17] Company, O., Pierrot, F., Shibukawa, T., and Morita, K. (2001) Four-Degree-of-Freedom Parallel Robot. European Patent, EP1084802, March 21, 2001.

[18] Suh, N. P. (2001). Axiomatic Design-Advances and Applications. Oxford University Press, 528 pp.
[19] El-Haik, B. and Yang, K. (1999). Measures of comlpexity in design. Proc. 1999 ASME Design Engineering Technical Conferences. Design Automation Conference, September 12-15, Las Vegas. Paper DETC99/DAC-8569.

[20] ElMaraghy, W.H. and Urbanic, J.R. (2003). "Modelling of manufacturing systems complexity," The Annals of CIRP, Vol. 53/1, pp. 363-366.

[21] Li, M. and Vitányi, P. (1997) An Introduction to Kolmogorov Complexity and Its Applications. Second Edition Springer Verlag, New York

[22] Wolfram, Stephen. (2002) A new Kind of Science, Wolfram Media Inc., Champaign, IL.

[23] Delahaye, J.-P. (1999) Information, complexité et hasard, Éditions Hermès, Paris.

[24] Delahaye, J.-P. (2003) "La complexité mesurée." Pour la science, No. 314, pp. 34-38.

[25] Wang, H. (1987). Reflections on Kurt Gödel, The MIT Press, Cambridge, MA.

[26] Angeles, J. and Morozov, A. (2003) A Device for the Production of Schönflies Motions. International Patent Application, filed Nov. 5, 2003.

[27] Angeles, J., Morozov, A., Slutski, L., Navarro, O., and Jabre, L., (2001). "The modular design of a long-reach, 11-axis manipulator." Proc. 2000 CISMIFToMM Symposium on Robots and Manipulators, Springer-Verlag, Vienna, pp. 225-233.

[28] Angeles, J., Morozov, A., and Navarro, O. (2000). “A novel manipulator architecture for the production of SCARA motions." Proc. IEEE Int. Conf. Robotics \& Automation, 2370-2375, San Francisco, April 24-28. CD-ROM 00CH37065C.

[29] Sutherland, G. and Roth, B., (1973). "A Tramsmission Index for Spatial Mechanisms", Journal of Engineering for Industry, pp.589-597.

[30] Al-Widyan, K., Angeles, J. and Ostrovskaya, S., (2003). "A robust simulation algorithm for conservative linear mechanical systems", International Journal for Multiscale Computational Engineering, Vol. 1, pp. 289-309. 